\title{
EAS thermal neutron detection with the PRISMA-LHAASO-16 experiment
}

\author{
S. H. Feng*, X. H. $\mathbf{M a}^{\dagger}$ \\ Key Laboratory of Particle Astrophysics, Institute of High Energy Physics, Chinese Academy of \\ Sciences \\ E-mail: maxheihep.ac.cn
}

Yu.V. Stenkin, O.B. Shchegolev

Institute for Nuclear Research, Russian Academy of Science

Danzengluobu, T. L. Chen, M. Y. Liu, Q. Gao

College of Science, Tibet University

S. W. Cui, Y. Y. He, B. B. Li

The College of Physics Science and Information Engineering, Hebei Normal University

\author{
R. Zhou, Q. C. Huang, J. D. Yao, F. Z. Shen
}

The College of Physical Science and Technology, Sichuan University

On behalf of the LHAASO Collaboration and the PRISMA Collaboration

\begin{abstract}
EAS thermal neutrons measurement gives additional valuable advantages to study energy and mass composition of primary cosmic rays especially above the knee region. After success of the PRISMA-YBJ experiment we built a new EAS thermal neutron detection array at Tibet University, Lhasa, China (3700m a.s.1.) in March, 2017. This prototype array consists of 16 EAS en-detectors measuring two main EAS components: hadronic and electromagnetic ones. These detectors use a thin layer of a novel type of $\mathrm{ZnS}(\mathrm{Ag})$ scintillator alloyed with natural boron compound for thermal neutron capture. In this report, we introduce principle of the detection technique, deployment of the array, and the test results obtained with the array.
\end{abstract}

35th International Cosmic Ray Conference - ICRC2017

10-20 July, 2017

Bexco, Busan, Korea

* Speaker.

$\dagger$ Speaker. 


\section{Introduction}

The cosmic ray energy spectrum spans over many decades from about $10^{6} \mathrm{eV}$ to beyond $10^{20}$ $\mathrm{eV}$. It consists of different regions with power law behavior and changes in the power law index. At about 3-5 $\times 10^{15} \mathrm{eV}$, there is a steepening of the spectrum, named the knee, which was measured by several experiments, e.g., KASCADE[四], Tibet AS $\gamma[\mathbb{[}]$ ], ARGO/WFCTA[B]], etc. Although the global features of the all-particle spectrum are reasonably recognized, the spectral shape of each primary component remains an open question related to the interpretation of the experimental data. In particular, high energy hadrons, which constitute the EAS skeleton, may carry important information for multi-parameter correlation studies, since some hadronic observables, primarily the hadron number/electron number correlation, depend on the nature of the particle inducing the shower [四][四]. Thus, the detection of high energy hadrons, addressed to improve the discrimination power in these analysis, is highly advisable. A way to deal with this problem avoiding the use of huge and expensive HCALs but using thermal neutron detectors, so called the PRISMA project (PRImary Spectrum Measurement Array), was brought out in [䧃] [ [ ] Thermal neutrons are generated abundantly from hadrons on the ground, up to 2 orders of magnitude more than parent hadrons. This idea led to the development of the EN-detector, made of a mixture of the well-known inorganic scintillator $\mathrm{ZnS}(\mathrm{Ag})$ with ${ }^{6} \mathrm{LiF}$, capable of recording both thermal neutrons and charged particles [ [U] [ [8]. The EN-detectors, relatively simple, compact and cheap, can be easily deployed in an air shower array to record simultaneously thermal neutrons and the charged particles in the shower front []. . A prototype array of 32 EN-detectors (PRISMA-32) is running in Moscow [Q] [ए人]].

In order to check the performance of the EN-detector at a high altitude site, a small array composed of four EN-detectors (PRISMA-YBJ) was installed inside the hall hosting the ARGO-YBJ experiment[W] at the Yangbajing Cosmic Ray Observatory (Tibet, China, $4300 \mathrm{~m}$ a.s.1.). Between ARGO-YBJ and PRISMA-YBJ, the coincident events generated by primary cosmic rays of energies greater than $100 \mathrm{TeV}$ were selected. The EN-detectors were used to record simultaneously thermal neutrons and the air shower electromagnetic component. The density distributions of both components and the total number of thermal neutrons were measured. The correlation of these data with the measurements carried out by ARGO-YBJ confirms the excellent performance of the EN-detector.[ए2]. Moreover, during long stable running, results on the natural thermal neutron flux are obtained. The existence of periodical variations both seasonal and related to the moon month are confirmed for the first time at high altitude. In particular, for synodic moon month the 4th harmonic with amplitude of about $0.5 \%$ and maximum with a delay of about 1 day after syzygies is found[[ए3]].

Because ${ }^{10} \mathrm{~B}$ is easier to be obtained than ${ }^{6} \mathrm{Li}$ and has similar specifications, a novel type of $\mathrm{ZnS}(\mathrm{Ag})$ scintillator alloyed with natural boron compound for thermal neutron capture is developed instead of $\mathrm{ZnS}\left(\mathrm{Ag}\right.$ ) with ${ }^{6} \mathrm{LiF}$, A prototype array of $72 \mathrm{ZnS}(\mathrm{Ag})$ with ${ }^{10} \mathrm{~B}_{2} \mathrm{O}_{3}$ EN-detectors so called "Upstairs array for Recoding of Atmospheric Neutrons" (URAN) is running in Moscow. In February 2017, we built a new array of $16 \mathrm{ZnS}(\mathrm{Ag})$ with ${ }^{10} \mathrm{~B}_{2} \mathrm{O}_{3}$ EN-detectors (PRISMA-LHAASO-16) at Tibet University (TU) in Lhasa, Tibet, China (3700 $\mathrm{m}$ a.s.1.) to check the performance of the new type EN-detectors at a high altitude site. Here we report the test results obtained with the array. 


\section{The Experimental Set-up}

In the PRISMA-LHAASO-16 experiment, the EN-detector is based on a special phosphor, which is a granulated alloy of inorganic $\mathrm{ZnS}(\mathrm{Ag})$ scintillator added with natural $\mathrm{B}_{2} \mathrm{O}_{3}$ with the ${ }^{10} \mathrm{~B}$ isotope about $20 \%$ (Fig. 四, left). One ${ }^{10} \mathrm{~B}$ captures one thermal neutron via the reaction:

$$
\begin{aligned}
{ }^{10} \mathrm{~B}+n \rightarrow 7 \mathrm{Li}^{*}+\alpha & \rightarrow{ }^{7} \mathrm{Li}+\alpha+0.48 \mathrm{MeV} \gamma+2.3 \mathrm{MeV}(93 \%) \\
& \rightarrow{ }^{7} \mathrm{Li}+\alpha+2.8 \mathrm{MeV}(7 \%)
\end{aligned}
$$

with cross section of 3980 barn. The phosphor is deposited in silicone rubber in the form of a thin one-grain layer. The scintillating compound grains used are of $0.3-0.8 \mathrm{~mm}$ in size. The effective thickness of the scintillator layer is $50 \mathrm{mg} / \mathrm{cm}^{2}$. Light yield of the scintillator is $\sim 160,000$ photons per neutron capture. The structure of a typical EN-detector is shown in Fig. $\square$, right. The scintillator of $0.36 \mathrm{~m}^{2}$ area is mounted inside a black cylindrical polyethylene (PE) 200-1 tank which is used as the detector housing. The scintillator is supported inside the tank to a distance of $30 \mathrm{~cm}$ from the photomultiplier (PMT) photocathode. A 4"-PMT (Beijing Hamamatsu CR-165) is mounted on the tank lid. A light reflecting cone made of foiled PE foam of 5-mm thickness is used to improve the light collection. As a result, $\sim 100$ photoelectrons per neutron capture are collected. The efficiency for thermal neutron detection in our scintillator was found experimentally by neutron absorption in the scintillator layer to be about $20 \%$. The peculiar characteristics of the EN-detector output, that are weak and fast signals from charged particles compared to high amplitude, slow and delayed signals from thermal neutron capture, make it well suitable for its use in the framework of EAS experiments[ए2].
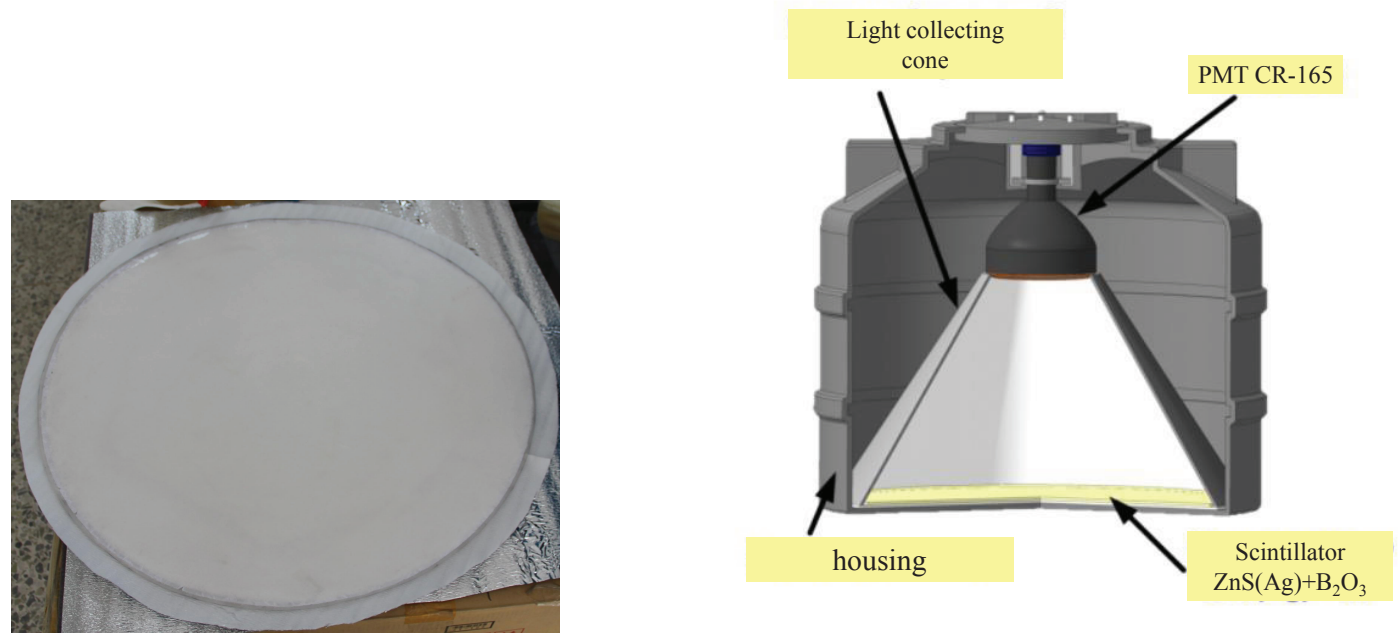

Figure 1: Left: Photo of the $\mathrm{ZnS}(\mathrm{Ag})+\mathrm{B}_{2} \mathrm{O}_{3}$ scintillator. Right: Scheme of the EN-detector.

In January 2017, the 16 EN-detectors were installed and tested at Hebei Normal University (HNU) (Fig. ఐ, left). In March, the PRISMA-LHAASO-16 array was installed at the top of the new building at Tibet University (TU). 15 of the EN-detectors are located in a $3 \times 5$ rectangle with distance of $5 \mathrm{~m}$ in east-west direction and $4.5 \mathrm{~m}$ in south-north direction, and the remained one is outside the rectangle (Fig. \, right).

A schematic view of the recording system of the array is shown in Fig. B]. The signal from 


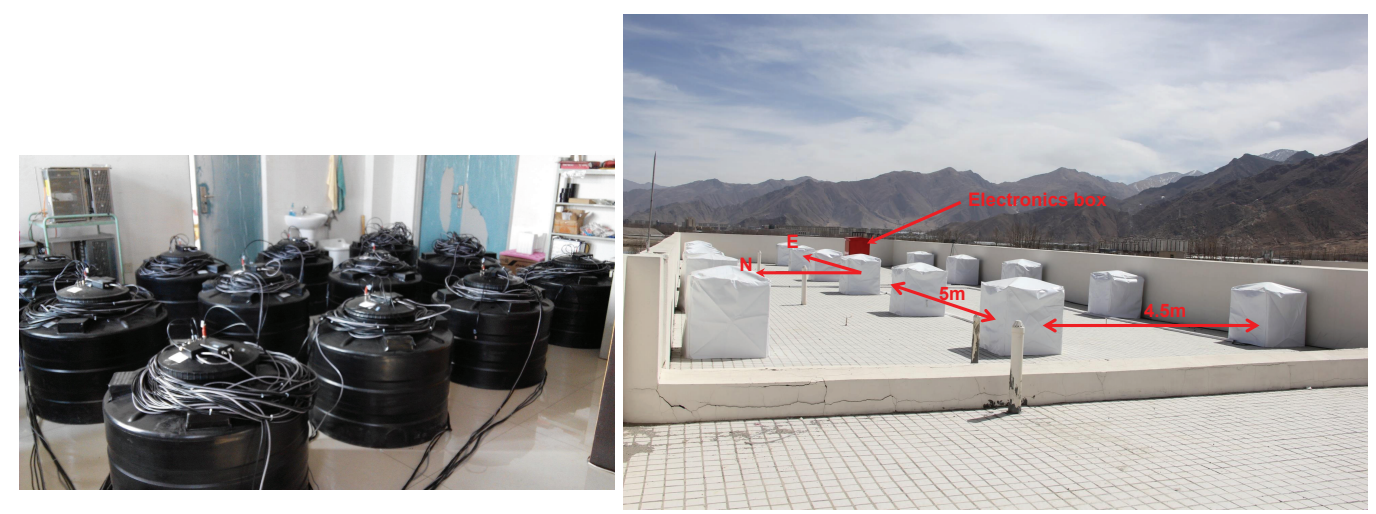

Figure 2: Left: Photo of the EN-detectors installed in the laboratory at HNU. Right: Photo of the ENdetector array at the top of one 4-floor building at TU.

the 8th dynode of each PMT is sent to a charge sensitive preamplifier-discriminator (DIU) where it is split into two pulses: one of them is shaped to a TTL pulse used to build-up the trigger, the other one is integrated with a $1 \mu \mathrm{s}$ time constant, then amplified and sent to the input of a FADC (made by Sichuan University, 32 channels, $14 \mathrm{bits}, 50 \mathrm{MS} / \mathrm{s}$ ). The signal from the 5 th dynode is sent to another charge sensitive preamplifier (UI) and then to the input of the FADC. The first pulse produced mostly by EAS electrons is used for trigger and energy deposit measurements, and delayed neutron capture pulses are counted within a time gate of $10 \mathrm{~ms}$ to give the number of neutrons. The first level trigger which is a coincidence of any 2 out of 16 detectors in a time gate of $1 \mu \mathrm{s}$, starts all FADC's. The on-line program analyzes the input data and provides the following second level triggers:

$\mathrm{M} 1$, if at least 2 detectors generating the first level trigger measured a signal corresponding to 10 mips or more;

$\mathrm{M} 2$, if the delivered total charge corresponds to more than 125 mips;

$\mathrm{M} 3$, if the total number of recorded neutrons is higher than or equal to 3 .

If any of these conditions is fulfilled thus the pulse shape of all signals is stored (20000 samples in steps of $0.5 \mu \mathrm{s}$.) along with the mark of the trigger number. In addition, every 5 minutes the on-line program generates a trigger (M0) which starts the data acquisition by the FADCs. This 'random' trigger, not related to showers, allows the measurement of chance signals which could mimic neutron signals in the $10 \mathrm{~ms}$ recording window.

\section{Data analysis and results}

The array has run for 4 months up to presents. Data are accumulated and the array is checked completely. Fig. $⿴$ is an example of big EAS events: some of detectors close to the shower core reach saturation at dynode 8 and has good record from dynode 5. Meanwhile, neutrons are measured at different distances from the shower core. With amount of events, correlation between dynode 8 and dynode 5 is obtained. For example, in Fig. [1, the left plot shows good linear correlation in one detector, and the right one shows saturation of dynode 8 in another detector. 


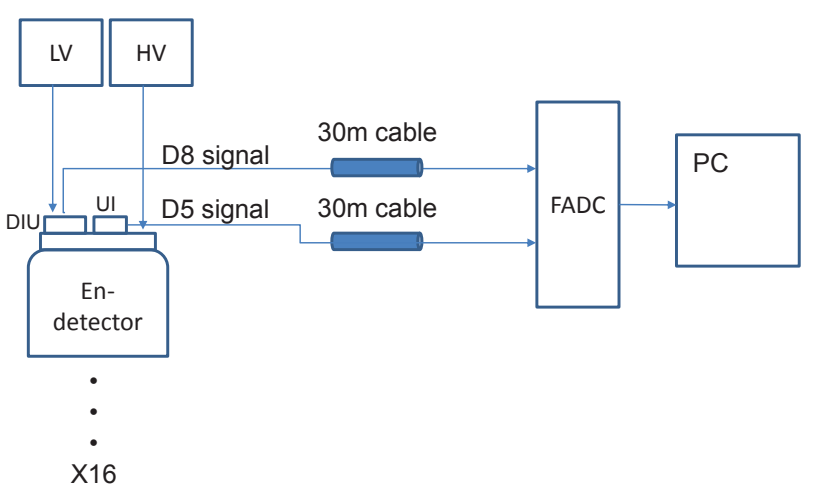

Figure 3: Scheme of the electronics system.

\section{Conclusions}

This paper is addressed to the test of the new type EN-detector in the framework of EAS measurements at high altitude. The sensitive layer of this detector is basically made of an alloy of an inorganic $\mathrm{ZnS}(\mathrm{Ag})$ scintillator alloyed with natural Boron with the ${ }^{10} \mathrm{~B}$ isotope. Its originality lies in simultaneously recording the air shower charged component and the thermal neutrons generated by high energy hadrons. The PRISMA-LHAASO-16 experiment is running smoothly and first preliminary results confirms that the EN-detectors worked properly at high altitude. It the first step to deploy the PRISMA units in the planned LHAASO array [14]]. The operation of the future large array of hundres of the EN-detectors located in the LHAASO experiment will enable the estimate of the hadron content in EAS, increasing capability of LHAASO to adequately determine the energy and nature of high energy cosmic rays.

\section{Acknowledgments}

This work is supported in China by National Natural Science Foundation (NSFC) (No.11375052, No.11563007, No.11463004) and the Research Project of Chinese Ministry of Education (No. 213036A), and in Russia by RFBR (grants 14-02-00996, 16-32-00054 and 16-29-13067_ofi_m), RAS Presidium Program "Fundamental properties of matter and astrophysics".

\section{References}

[1] T. Antoni et al. Astroparticle Physics 24 (2005) 1.

[2] M. Amenomori et al. Physics Letters B 632 (2006) 58.

[3] B. Bartoli et al. Physical Review D 92 (2015) 092005 

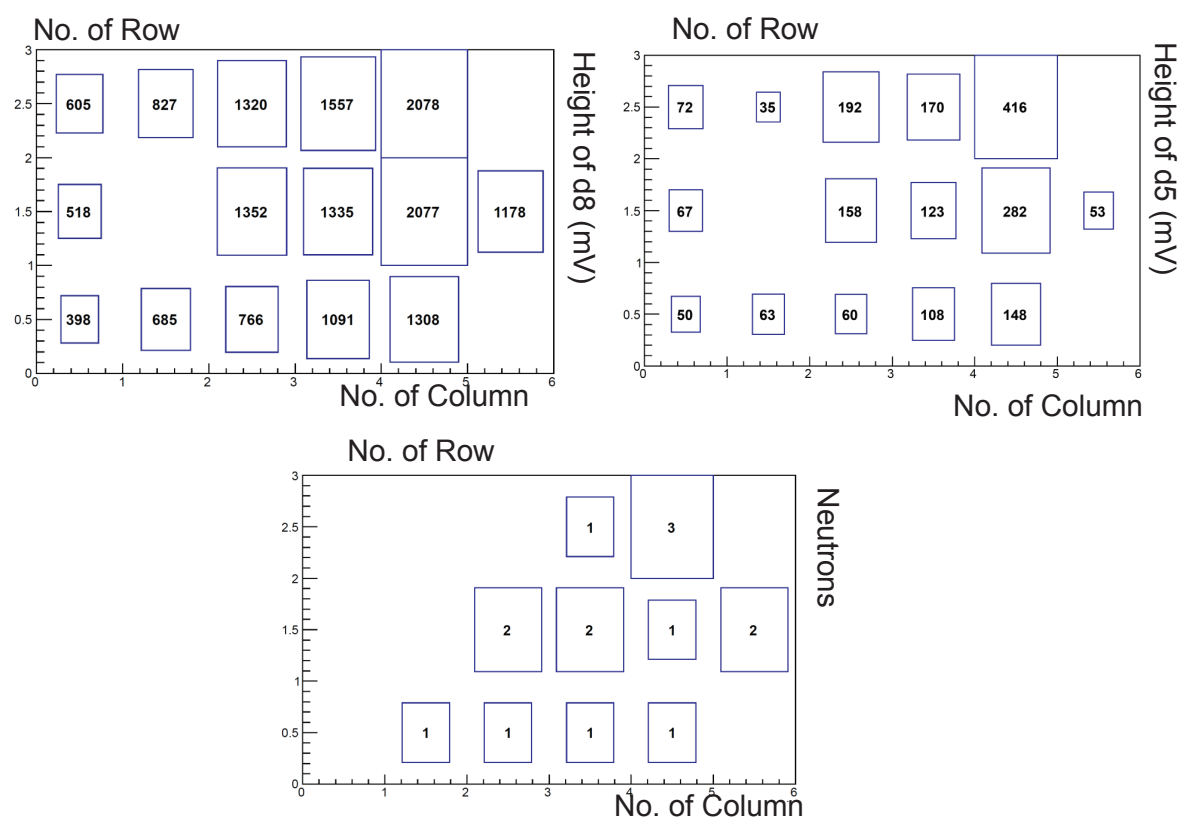

Figure 4: One real EAS event detected by the array. One detector per bin. Upper left: Pulse height distribution of dynode 8. Upper right: Pulse height distribution of dynode 5. Lower: Neutrons distribution.

[4] Yu.V. Stenkin and J.F. Valdés-Galicia, Modern Physics Letters A 17(26) (2002) 1745.

[5] Yu.V. Stenkin. Nuclear Physics B (Proc. Suppl.) 196 (2009) 293.

[6] Yu.V. Stenkin and J.F. Valdés-Galicia. Proceedings of 27th International Cosmic Ray Conference, Hamburg (2001) 1453.

[7] Yu.V. Stenkin, D. D. Djappuev, and J. F. Valdés-Galicia. Physics of Atomic Nuclei, 70(6) (2007) 1088.

[8] Yu.V. Stenkin. Nuclear Physics B (Proc. Suppl.), 175-176 (2008) 326.

[9] Yu.V. Stenkin, et al. Proceedings of 32nd International Cosmic Ray Conference, Beijing, (2011) ID: 1136.

[10] D. Gromushkin, et al. Journal of Instrumentation, 9 (2014) C08028.

[11] B. D'Ettorre Piazzoli on behalf of the ARGO-YBJ collaboration, Proceedings of 32nd International Cosmic Ray Conference, Beijing, 12 (2011) 93.

[12] B. Bartoli et al. Detection of Thermal Neutrons with the PRISMA-YBJ Array in Extensive Air Showers Selected by the ARGO-YBJ Experiment, Astroparticle Physics 81 (2016) 49íC60.

[13] Y. Stenkin et al. Seasonal and Lunar Month Periods Observed in Natural Neutron Flux at High Altitude, Pure Appl. Geophys. (2017). doi:10.1007/s00024-017-1545-7

[14] Z. Cao, Chinese Phys. C 34(2) (2010) 249. 

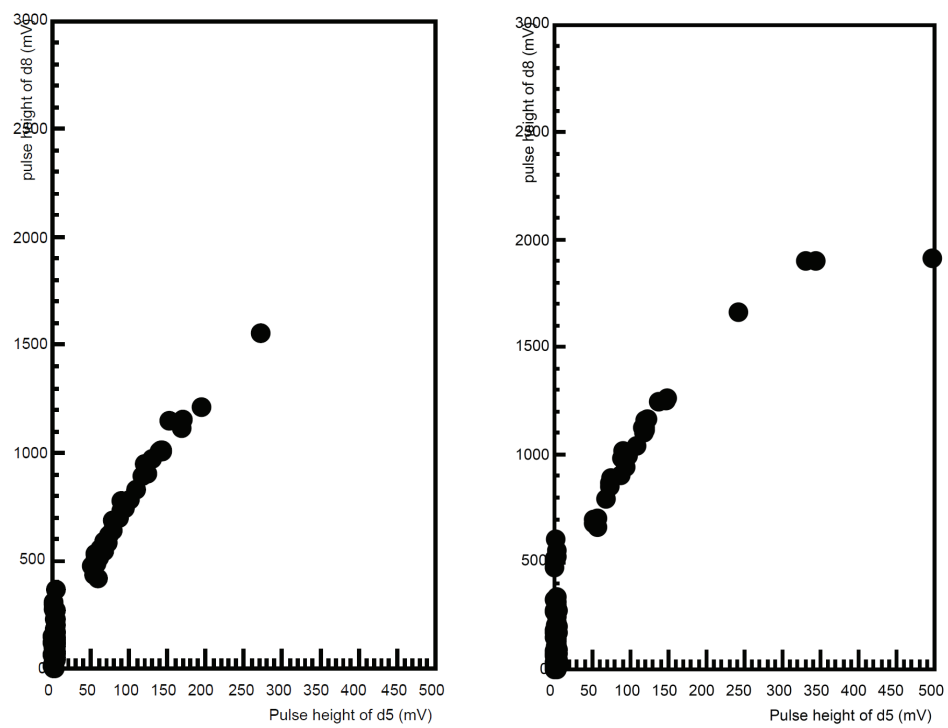

Figure 5: Pulse height of dynode 8 vs 5 of two detectors. 